\title{
Laboratory rearing methods that promote inbreeding have a negative impact on the fitness of Mastrus ridens Horstmann (Hymenoptera: Ichneumonidae), a parasitoid used to control the codling moth
}

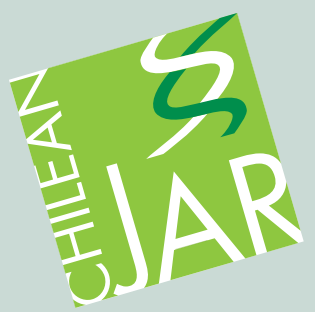

Erick Bueno ${ }^{1}$, Alda Romero ${ }^{1}$, Iván Osorio ${ }^{1}$, and Tania Zaviezo ${ }^{1 *}$

\section{ABSTRACT}

Classical and augmentation biological control depend on the successful laboratory rearing of natural enemies, but rearing methods can affect the success and productivity of a laboratory colony. One process that can occur during rearing is the loss of genetic variability due to inbreeding. The aim of this study was to examine the influence of rearing methods with and without inbreeding in Mastrus ridens Horstmann, a hymenopteran parasitoid specialist of the codling moth (Cydia pomonella [L.]), on several fitness traits. Traits of females from mixed and endogamous populations resulting from different rearing methods were compared, as well as traits of females from the same population subjected to sib-mating or outbreeding. Longevity, parasitism, total fecundity, and number of sons were similar for females in both evaluations. The number of daughters was lower $(1.9 \pm 0.6$ $v s .4 .5 \pm 0.5)$ and the offspring sex ratio was higher $(0.81$ \pm 0.04 vs. $0.48 \pm 0.05$ proportion of males) in females from the endogamous population compared to females of the mixed population, as well as in sib-mating females compared to outbred females of the same population $(0.5$ \pm 0.3 vs. $5.8 \pm 1.9$ daughters and $0.94 \pm 0.03$ vs. $0.51 \pm$ 0.10 sex ratio). These results show that rearing methods, specifically inbreeding conditions, have an effect on $M$. ridens fitness; thus, successful outbred laboratory rearing should be promoted.

Key words: Biological control, longevity, parasitism, rearing, sex ratio.

${ }^{1}$ Pontificia Universidad Católica de Chile, Facultad de Agronomía e Ingeniería Forestal, Casilla 306-22, Santiago, Chile.

"Corresponding author (tzaviezo@uc.cl).

Received: 7 June 2017.

Accepted: 6 October 2017

doi:10.4067/S0718-58392017000400413

\section{INTRODUCTION}

Biological control is the use of living organisms (usually referred to as natural enemies or biological control agents) to suppress pest populations to acceptable levels (Van Driesche et al., 2009; van Lenteren, 2012). Biological control is an important control strategy in integrated pest management (IPM) and one of the main alternatives that is compatible with organic agriculture. There are three approaches to biological control (Van Driesche et al., 2009; Heimpel and Mills, 2017): (a) classical or importation biological control in which natural enemies from the native range of the pest are brought into a new area, (b) augmentation biological control in which natural enemies are mass-reared and then released in a programmed manner, and (c) conservation biological control in which the crop and environment are managed to increase the survival of natural enemies, permanence, or immigration, so they can increase their impact on the pest.

Both classical and augmentation biological control depend on successful laboratory rearing of natural enemies, but initial population composition, rearing methods, and time in captivity can affect the success of a laboratory colony (Woodworth et al., 2002; Reed et al., 2003; Gilchrist et al., 2012; Francuski et al., 2014), and ultimately their success in controlling the pest once released in the field (Taylor et al., 2011; Fowler et al., 2015). One process that might occur during the rearing of natural enemies is inbreeding, which can lead to inbreeding depression, that is, decreased fitness of the offspring of genetically related parents (Charlesworth and Charlesworth, 1987; Charlesworth and Willis, 2009). Among the natural enemies, parasitoids of the order Hymenoptera have been extensively used in both classical and augmentation biological control programs (Heimpel and Lundgren, 2000; Wajnberg et al., 2008; van Lenteren, 2012; Heimpel and Mills, 2017). An interesting feature of most parasitic Hymenoptera is their haplodiploid mode of sex determination where males develop from unfertilized eggs (haploids) and females are produced from fertilized eggs (diploids) (Heimpel and de Boer, 2008). Haplodiploid species generally exhibit lower inbreeding depression than diploid species because many harmful alleles can be purged via males (Henter, 2003). Nevertheless, from insignificant to considerable inbreeding effects have been found on hymenopteran parasitoid species (Antolin, 1999). An additional consideration is related to species with complementary sex determination (CSD) in which the allelic composition at the sex locus (or loci) is decisive. In these species, haploid individuals develop into males and diploid individuals develop into females if 
they are heterozygote or into males if they are homozygote (Heimpel and de Boer, 2008; Ode and Hardy, 2008). Thus, inbreeding in these species increases the chances of producing diploid males and because these males in almost all species are less viable, sterile, or produce non-viable daughters, their production can negatively impact population growth, particularly when numbers are low (Zayed and Packer, 2005; Fauvergue et al., 2015).

Augmentation of natural enemy releases requires mass rearing of large numbers of good quality individuals in the most inexpensive way, especially in commercial settings (van Lenteren, 2012). Important aspects of the biology of the species must be known to achieve this, particularly those traits that most directly affect rearing productivity and their impact on the pest once released, that is, fitness traits, particularly those that maximize lifetime reproductive success. Longevity, parasitism success, fecundity, and offspring sex ratio are among the most important traits related to fitness in rearing for hymenopteran parasitoids. In the field, traits associated with dispersal and host searching also become crucial (van Baalen and Hemerik, 2008). Offspring sex ratio and daughter production are very important in parasitoids because females are responsible for attacking the host, increasing population numbers, and successfully controlling the pest.

Mastrus ridens Horstmann (Hymenoptera: Ichneumonidae) is an important parasitoid of the codling moth (Cydia pomonella [L.]) in its region of origin (southern Kazakhstan and northwestern China) (Mills, 2005; Retamal et al., 2016). It is a specialist gregarious ectoparasitoid that attacks mature codling moth larvae in their cocoons; it has been used in classical biological control programs against this pest in several countries (Mills, 2005; Devotto et al., 2010; Sandanayaka et al., 2011a; Tortosa et al., 2014) and has the potential for use in augmentation programs. Studies carried out in our laboratory indicate that this species displays CSD; colonies with a longer rearing history and more transfers had lower genetic diversity and produced a larger proportion of diploid males (Retamal et al., 2016). The aim of this study was to examine the influence of $M$. ridens rearing methods, specifically those concerning inbreeding, on several fitness traits to optimize rearing. We hypothesized that inbred females will have lower fitness than outbred females and that inbreeding will result in a larger proportion of male offspring.

\section{MATERIALS AND METHODS}

\section{Insect origin and rearing}

Mastrus ridens populations have been maintained in the laboratory since 2013. Insects forming these populations are individuals descending from field collections made near Almaty, Kazakhstan in 2013 and 2015, and individuals from the mass rearing of the New Zealand colony that were imported to the laboratory in 2014. Insects from the New Zealand colony are descendants of $M$. ridens collected in the same area in the 1990s by Mills and collaborators
(Mills, 2005; Retamal et al., 2016). To maintain $M$. ridens populations, mated females were provided with Cydia pomonella cocooned fifth instar larvae on pieces of corrugated cardboard, which were replaced every 3 to $7 \mathrm{~d}$ with cardboard containing fresh cocooned larvae. Females were provided with a streak of organic honey as food. Environmental conditions were 20 to $25^{\circ} \mathrm{C}, 40 \%$ to $70 \%$ $\mathrm{RH}$, and 16:8 h photoperiod.

The host, $C$. pomonella, was reared on an artificial diet (Stonefly Heliothis Diet; Ward's Natural Science, Rochester, New York, USA) at 21 to $25{ }^{\circ} \mathrm{C}, 20 \%$ to $60 \%$ $\mathrm{RH}$, and 16:8 h photoperiod. After 17 to $24 \mathrm{~d}$ on the diet, larvae were removed and placed on pieces of corrugated cardboard in which they readily spun a cocoon. These cocooned larvae were either exposed to the parasitoids or allowed to pupate and continue the host colony. For the latter, groups of 30 to 40 codling moth pupae were placed in oviposition cages consisting of cylinders, $25 \mathrm{~cm}$ diameter and $80 \mathrm{~cm}$ length, lined on the inside with wax paper used as oviposition substrate. When adult moths emerged, they were fed with diluted honey and maintained in the oviposition cages for 2 to $3 \mathrm{wk}$.

\section{Fitness of females from populations under rearing methods with or without inbreeding}

To study the effects of laboratory rearing methods on $M$. ridens fitness, several traits of females from populations that differed in handling protocols were compared. The mixed population (MIX) was composed of insects from different origins (i.e., descendants of the 2013 and 2015 field collections as well as the New Zealand colony), that were handled in small cages $(15 \times 23 \times 4 \mathrm{~cm})$ containing eight females and four males that were provided with host larvae every 4 to $7 \mathrm{~d}$ according to availability. After 2 wk, when offspring were in the pupal stage, initial $M$. rindes adults were removed and cages were monitored daily for adult emergence. Outbreeding was promoted by mixing newly emerged females in a given cage and males originating from different cages. However, for fitness traits measurements, females had to be individually monitored. A sample of females was collected from the cages and they were allowed to individually oviposit in small glass vials $(2.5 \times 5.5 \mathrm{~cm})$. When their offspring reached the pupal stage, they were isolated in small vials and emerging females were mated with non-brothers, which were then provided with host larvae and allowed to oviposit until death. This procedure was carried out with 15 females in four consecutive generations $(\mathrm{N}=60)$.

The endogamous populations (ENDO) corresponded to iso-female lines, that is, originating from only one female, which were initiated by mating a female of the MIX population with a brother (sib-mating). Mated females were individually handled in small glass vials $(2.5 \times 5.5$ $\mathrm{cm})$ to which host larvae were added. Two weeks after the beginning of oviposition, host larvae were checked for parasitization and, if present, offspring pupae were placed in small glass vials until adult emergence. From then on, sib- 
mating was repeated in each generation. For comparative evaluations, females came from two iso-female lines that have been maintained for over 10 mo under this rearing method. Fitness traits were assessed on six females from each line and in four consecutive generations $(\mathrm{N}=42)$.

The fitness traits measured for females from the MIX and ENDO populations were (a) longevity of reproducing females: days from adult emergence to death; (b) percentage parasitism: number of parasitized larvae divided by total number of larvae exposed to female in its lifetime; (c) fecundity: total emerged adult offspring (lifetime number of sons and daughters produced by a female), and (d) offspring secondary sex ratio: lifetime number of sons divided by total emerged adult offspring. Evaluated insects were maintained under the same environmental conditions $\left(25 \pm 3{ }^{\circ} \mathrm{C} ; 50 \pm 10 \% \mathrm{RH}, 16: 8 \mathrm{~h}\right.$ photoperiod $)$, host larvae were exposed to females at a rate of one per day, and a streak of organic honey was provided as food for adult parasitoids.

\section{Fitness of females from the same population reared with and without inbreeding}

As a complement, we compared fitness traits of $M$. ridens females from the same population but handled differently for mating. The population used in this experiment included the descendants of the 2015 field collections (KZ15) that were maintained under culture for approximately 4 mo before the experiment. At the beginning, field-collected insects were handled by placing individual females in glass vials, but insects were handled in groups as numbers increased, similar to what was described for the MIX population. However, for fitness trait measurements and controlled mating, females were individually monitored using the previously described procedures. For this experiment, emerging females were placed in a vial with either a brother (sib-mating; $\mathrm{N}=18$ ) or a male from the MIX population (outbreeding; $\mathrm{N}=10$ ), and were allowed to oviposit after $24 \mathrm{~h}$. The fitness traits measured and experimental conditions were the same as those mentioned above.

\section{Data analysis}

Generalized linear models (GLM) were used to test the effect of the population rearing method (MIX or ENDO), generation (1 to 4), and their interaction on female fitness traits. The best-fitted models used a normal distribution with an identity link function for count data (fecundity and longevity) and binomial distribution with a logit link function for proportional data (parasitism and sex ratio). Initial analyses indicated that females from the two different iso-female lines did not differ in any of the fitness traits measured (GLM probabilities for population effect from 0.44 to 0.96 ), so they were combined into one category (ENDO) for the final analyses. Similarly, GLMs were used to test the effect of mating type (sib-mating or outbreeding) on fitness traits of females from the same population (KZ15). The GLM models were analyzed with the JMP 13 statistical software (SAS Institute, Cary, North Carolina, USA). All the means in the text and figures are followed by \pm 1 standard error (SE).

\section{RESULTS}

Fitness of females from populations under rearing methods with or without inbreeding

Longevity was similar for females of both populations (GLM: $\chi_{1,7}^{2}=0.88, \mathrm{P}=0.35$; Figure $\left.1 \mathrm{~A}\right)$. There was some variation in female longevity among generations (GLM: $\chi_{3,7}^{2}=10.12$, $\mathrm{P}=0.02)$, and it was lower in the first generation than in the third $(16.2 \pm 1.6 \mathrm{vs} .22 .7 \pm 1.6 \mathrm{~d})$. There was also a population $\times$ generation effect $\left(\mathrm{GLM}: \chi_{3,7}^{2}=7.99, \mathrm{P}=0.046\right)$ and first-generation females from the MIX population lived longer than females from the ENDO population $(19.1 \pm 1.6$ vs. $11.8 \pm 1.5 \mathrm{~d})$, while the opposite occurred in the second generation $(20.3 \pm 3.3 \mathrm{vs} .15 .4 \pm 1.0 \mathrm{~d})$.

Females from both populations showed similar parasitism (GLM: $\chi_{1,7}^{2}=0.81, \mathrm{P}=0.37$; Figure $1 \mathrm{~B}$ ) with an overall mean of $32.3 \pm 2.4 \%$ parasitized larvae. There was no effect for generation (GLM: $\chi_{3,7}^{2}=1.45, \mathrm{P}=0.69$ ) or population $\times$ generation interaction (GLM: $\left.\chi_{3,7}^{2}=4.44, \mathrm{P}=0.22\right)$.

Female fecundity, measured as lifetime emerged adult offspring, was on average $9.3 \pm 0.8$ offspring per female, consisting of $6.0 \pm 0.6$ sons and $3.4 \pm 0.4$ daughters. Fecundity was similar for females from both populations (GLM: $\chi_{1,7}^{2}=0.70, \mathrm{P}=0.40$; Figure $1 \mathrm{C}$ ) and for generations (GLM: $\chi_{3,7}^{2}=3.55, \mathrm{P}=0.31$ ), but there were differences between populations in the first two generations (GLM: $\chi_{3,7}^{2}=14.78, \mathrm{P}=0.002$ ). Offspring numbers in the first generation were almost twice the number of offspring in the ENDO population compared to the MIX population $(13.9 \pm 3.9 v s .7 .1 \pm 1.6$ offspring $)$, but this was reversed in the second generation $(5.4 \pm 1.7 v s .15 .5 \pm 2.6$ offspring $)$. The observed pattern for fecundity was mainly driven by the number of sons, which showed the same population $\times$ generation effect (GLM: $\left.\chi_{3,7}^{2}=17.99, \mathrm{P}<0.001\right)$. On the other hand, the number of daughters differed between populations (GLM: $\chi_{1,7}^{2}=9.79, \mathrm{P}=0.002$; Figure 1C). Females from the MIX population produced $4.5 \pm 0.5$ daughters over a lifetime, while females from the ENDO population produced only $1.9 \pm 0.6$ daughters. No effects of generation or population $\times$ generation were found $(\mathrm{P}>0.45$ for both). Of those females that successfully parasitized host larva, only $63 \%$ produced daughters in the ENDO population compared to $76 \%$ in the MIX population.

The offspring sex ratio was biased toward males in the ENDO population ( $81 \%$ sons), but this was not so in the MIX population (48\% sons) (GLM: $\chi_{1,7}^{2}=9.76, \mathrm{P}=0.002$; Figure 1D). No generation or population $\times$ generation effects were found ( $\mathrm{P}>0.68$ for both).

Fitness of females from the same population reared with and without inbreeding

Mating type, sib-mating or outbreeding, did not influence female longevity (GLM: $\chi_{1,1}^{2}=1.34, \mathrm{P}=0.25$; Figure $2 \mathrm{~A}$ ) 
Figure 1. Fitness traits of female Mastrus ridens from populations with different rearing methods (mixed, MIX and endogamous, ENDO) across four generations. (A) Longevity of reproducing females (d), (B) percentage parasitism (total number of parasitized larvae from the total number of exposed larvae), (C) fecundity (lifetime total emerged adult progeny; sons at the bottom of bars with striped pattern; daughters at top of bars with solid pattern), and (D) lifetime offspring sex ratio (proportion of males).
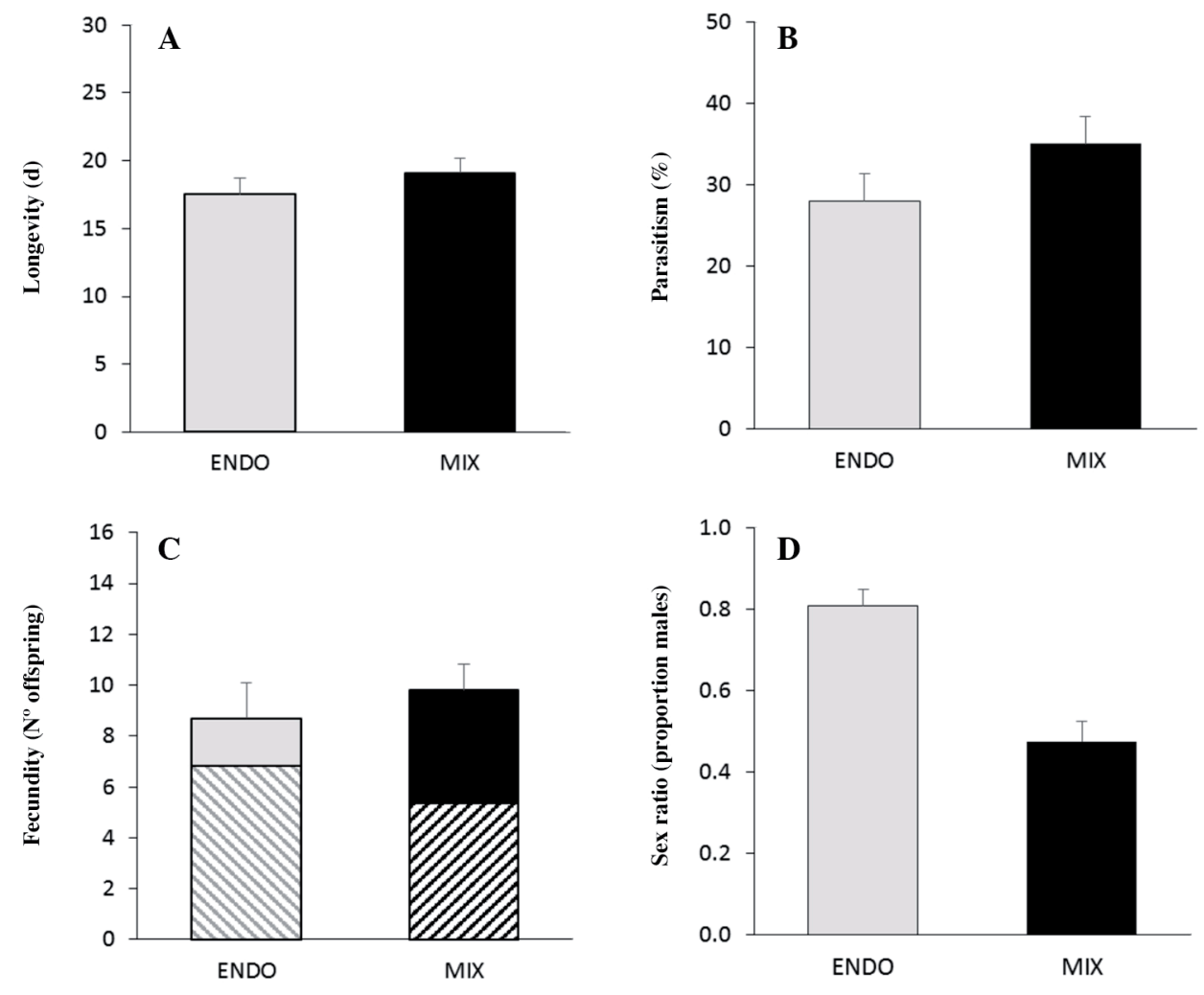

Error bars represent standard errors (SE).

or parasitism rate (GLM: $\chi_{1,1}^{2}=0.89, \mathrm{P}=0.35$; Figure $\left.2 \mathrm{~B}\right)$. On average, sib-mated females produced $6.5 \pm 1.9$ offspring in their lifetime, and outbred females $11.1 \pm 2.7$ offspring (Figure 2C), but these differences were nonsignificant (GLM: $\left.\chi_{1,1}^{2}=2.11, \mathrm{P}=0.15\right)$. The sib-mated and outbred females produced a similar number of sons (GLM: $\chi_{1,1}^{2}=$ $0.08, \mathrm{P}=0.78$; Figure $2 \mathrm{C}$ ), but sib-mated females produced only $0.5 \pm 0.3$ daughters while outbred females had $5.8 \pm$ 1.9 daughters in their lifetime (GLM: $\chi_{1,1}^{2}=12.10, \mathrm{P}<$ 0.001 ; Figure 2C). The low numbers of daughters produced by the sib-mated females were partly explained by the fact that only $34 \%$ of females that successfully parasitized had daughters, while this was $64 \%$ in outbred females. The offspring sex ratio was heavily biased toward males in sibmated females (94\%), while approximately half of the offspring of outbred females were males (GLM: $\chi_{1,1}^{2}=$ 5.08, $\mathrm{P}=0.02$; Figure 2D).

\section{DISCUSSION}

In our study, we examined the influence of rearing methods, particularly with and without inbreeding, on several $M$. ridens fitness traits, a specific parasitoid of the codling moth, to gain knowledge that could optimize its rearing. Earlier studies with this parasitoid species have looked at several aspects of laboratory rearing methods on several $M$. ridens fitness traits, including the effect of temperature, food type for adults, mating, larval host weight, host availability, and parasitoid to host ratios (Bezemer and Mills, 2003; Devotto et al., 2010; Sandanayaka et al., 2011a; 2011b).

The results of our study show that rearing methods that promote inbreeding in the laboratory only have an impact on some $M$. ridens fitness traits. Longevity of adult reproducing females, with access to honey as food, lived on average 18.5 to $19.5 \mathrm{~d}$ under experimental conditions; longevity was not affected by inbreeding because females from the MIX and ENDO populations or sib-mated and outbred females from the KZ15 population had similar longevity. The number of days that females lived in this experiment was similar to findings reported in earlier studies at comparable temperatures (Bezemer and Mills, 2003; Devotto et al., 2010; Sandanayaka et al., 2011b).

Parasitism by females from the MIX and ENDO populations was similar (overall mean $32 \%$ ), as well as that of sib-mated and outbred females from the KZ15 population (overall mean 18\%). Parasitism rates observed in the present study were also within the range of earlier studies. Bezemer and Mills (2003) reported 13\% parasitism under laboratory conditions and $17 \%$ in the field in California, USA. Sandanayaka et al. (2011a) reported 29\% parasitism in the New Zealand laboratory colony when single females were presented with 10 host larvae. 
Figure 2. Fitness traits of female Mastrus ridens from the same population and different rearing methods (sib-mating and outbreeding) across four generations. (A) Longevity of reproducing females (d), (B) percentage parasitism (total number of parasitized larvae from total number of exposed larvae), (C) fecundity (lifetime total emerged adult progeny; sons at the bottom of bars with striped pattern; daughters at top of bars with solid pattern), and (D) lifetime offspring sex ratio (proportion of males).
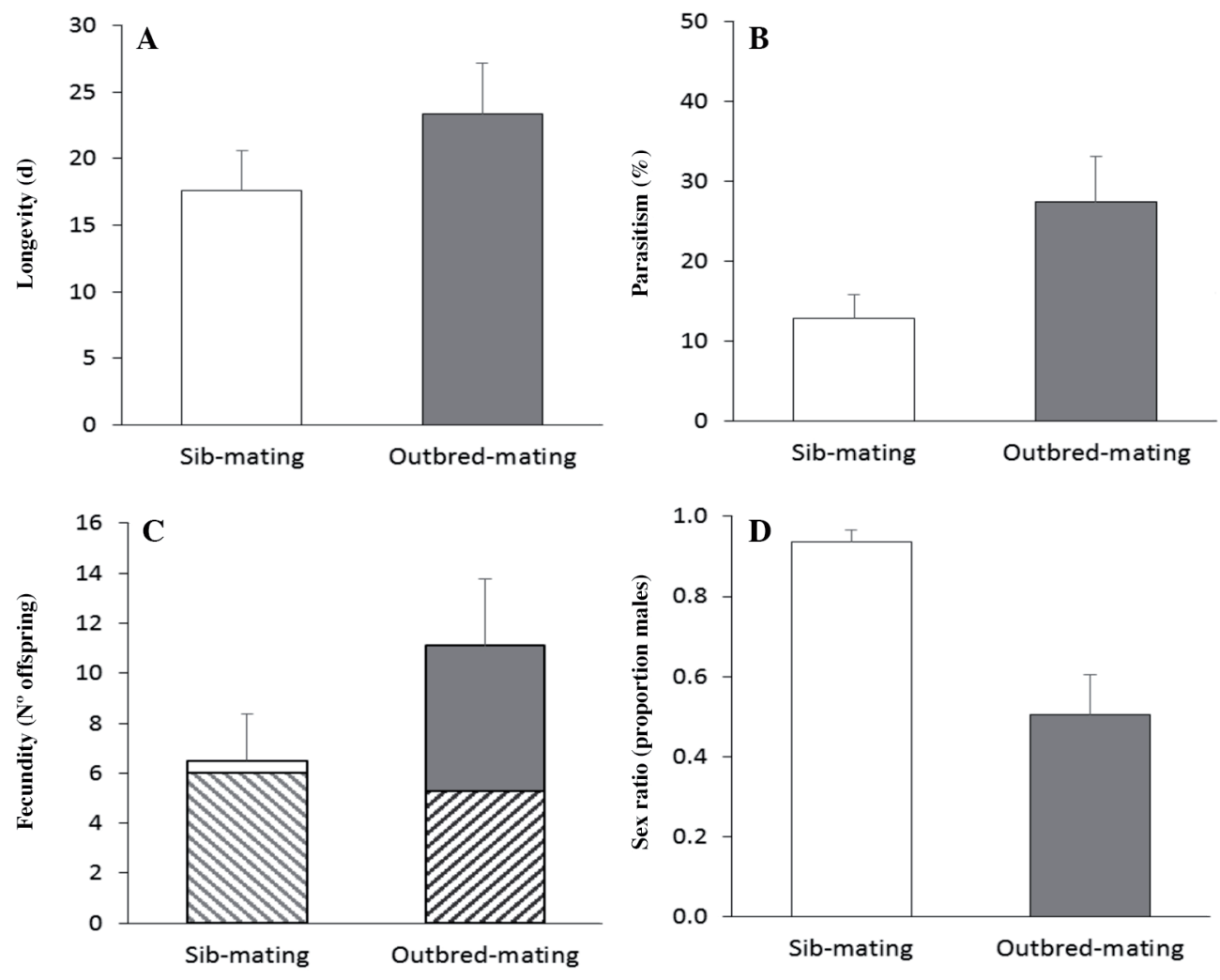

Error bars represent standard errors (SE).

Female fecundity, that is, lifetime emerged adult offspring and number of sons were not significantly different for females from the MIX and ENDO populations, nor for the sib-mated and outbred females from the KZ15 population. Even though we did not find significant differences in the number of sons in the comparative evaluations, there was a trend toward more sons in the progeny of females with inbreeding (ENDO and sib-mated KZ15); perhaps increasing experimental numbers would have allowed significant differences to be detected. One surprising result was the low lifetime fecundity found in our populations, which was less than half the values reported by Bezemer and Mills (2003) and Devotto et al. (2010). One likely explanation for these differences might be our use of non-diapausing host larvae, while the other two mentioned studies used diapausing larvae. Therefore host larvae in our study were less time susceptible and parasitoid females had less time to attack the larvae. This is because the nondiapausing larvae continued their development and could reach a stage where $M$. ridens does not develop. In fact, among the larvae exposed to $M$. ridens in our experiments, between $17 \%$ and $50 \%$ were able to reach the pupal stage (data not shown).

The number of daughters was consistently lower in the inbreeding treatments when compared to outbreeding treatments, which resulted in a heavily male-biased offspring sex ratio ( $>81 \%$ sons in the progeny) for the ENDO population and sib-mated KZ15 population. Given that $M$. ridens is a haplodiploid species with CSD, the most probable cause of this high male-biased sex ratio would be the production of diploid sons instead of daughters (Heimpel and Lundgren, 2000; Heimpel and de Boer, 2008; Ode and Hardy, 2008; de Boer et al., 2015). Heimpel and Lundgren (2000) studied sex ratios in commercially reared parasitoids and found several cases of male-biased sex ratios, and they attributed this to inbreeding in most circumstances. By reviewing experimental studies of inbreeding depression in haplodiploid species, Antolin (1999) found no changes in sex ratio in species without CSD. The sex ratio was not reported in the one species with CSD that was included in the review, but male sterility increased with inbreeding, suggesting diploid male production (Antolin, 1999).

More detailed and controlled studies of the effects of inbreeding in $M$. ridens are currently being carried out to understand if the production of diploid males, and their reproductive capabilities compared to haploid males, could be responsible for the observed differences in the number of daughters and sex ratios produced by females under inbred and outbred rearing protocols. 


\section{CONCLUSIONS}

The results of this study show that rearing methods, specifically inbreeding conditions, have an effect on Mastrus ridens fitness. Not all measured traits were negatively affected by inbreeding, but the number of female offspring was lower and the offspring sex ratio (proportion of males) was higher under inbreeding. These two traits are particularly important when developing efficient mass rearing, and they are also key quality attributes of released parasitoids to maximize pest control. Therefore, laboratory rearing methods in $M$. ridens should promote outbreeding.

\section{ACKNOWLEDGEMENTS}

This study was financed by the Chilean Government through the FONDECYT 1131145 project. We are grateful to Kazbek Toleubayev (The Kazakh Research Institute for Plant Protection and Quarantine) for facilitating field collections, Manoharie Sandanayaka (Plant and Food Research, New Zealand) for the importation of insects from their colony, and to many students who helped to maintain the insect colonies.

\section{REFERENCES}

Antolin, M.F. 1999. A genetic perspective on mating systems and sex ratios of parasitoid wasps. Researches on Population Ecology 41:29-37.

Bezemer, T.M., and Mills, N.J. 2003. Clutch size decisions of a gregarious parasitoid under laboratory and field conditions. Animal Behaviour 66:1119-1128.

Charlesworth, D., and Charlesworth, B. 1987. Inbreeding depression and its evolutionary consequences. Annual Review of Ecology and Systematics 18:237-268.

Charlesworth, D., and Willis, J.H. 2009. The genetics of inbreeding depression. Nature Reviews Genetics 10:783-796.

De Boer, J.G., Groenen, M.A.M., Pannebakker, B.A., Beukeboom, L.W., and Kraus, R.H.S. 2015. Population-level consequences of complementary sex determination in a solitary parasitoid. BMC Evolutionary Biology 15:98.

Devotto, L., del Valle, C., Ceballos, R., and Gerding, M. 2010. Biology of Mastrus ridibundus (Gravenhorst), a potential biological control agent for area-wide management of Cydia pomonella (Linneaus) (Lepidoptera: Tortricidae). Journal of Applied Entomology 134:243-250.

Fauvergue, X., Chuine, A., Vayssade, C., Auguste, A., and Desouhant, E. 2015. Sterile males in a parasitoid wasp with complementary sex determination: from fitness costs to population extinction. BMC Ecology 15:13.

Fowler, S.V., Paterson, P., Barret, D.P., Forgie, S., Gleeson, D.M., Harmann, H., et al. 2015. Investigating the poor performance of heather beetle, Lochmaea suturalis (Thompson) (Coleoptera: Chrysomelidae), as a weed biocontrol agent in New Zealand: has genetic bottlenecking resulted in small body size and poor winter survival? Biological Control 87:32-38.

Francuski, L., Djurakic, M., Ludoski, J., Hurtado, P., Pérez-Bañón, C., Stahls, G., et al. 2014. Shift in phenotypic variation coupled with rapid loss of genetic diversity in captive populations of Eristalis tenax (Diptera: Syrphidae): consequences for rearing and potential commercial use. Journal of Economic Entomology 107:821-832.
Gilchrist, A.S., Cameron, E.C., Sved, J.A., and Meats, A.W. 2012. Genetic consequences of domestication and mass rearing of pest fruit fly Bactrocera tryoni (Diptera: Tephritidae). Journal of Economic Entomology 105:1051-1056.

Heimpel, G.E., and de Boer, J.G. 2008. Sex determination in the hymenoptera. Annual Review of Entomology 53:209-230.

Heimpel, G.E., and Lundgren, J.G. 2000. Sex ratios of commercially reared biological control agents. Biological Control 19:77-93.

Heimpel, G.E., and Mills, N.J. 2017. Biological control: Ecology and applications. 386 p. Cambridge University Press, Cambridge, UK.

Henter, H.J. 2003. Inbreeding depression and haplodiploidy: experimental measures in a parasitoid and comparisons across diploid and haplodiploid insect taxa. Evolution 57:1793-1803.

Mills, N.J. 2005. Selecting effective parasitoids for biological control introductions: Codling moth as a case study. Biological Control 34:274-282.

Ode, P.J., and Hardy, I.C.W. 2008. Parasitoid sex ratios and biological control, p. 253-291. In Wajnberg, E., Bernstein, C., and van Alphen, J. (eds.) Behavioral ecology of insects parasitoids: From theoretical approaches to field applications. Blackwell Publishing, Malden, Massachusetts, USA.

Reed, D.H., Lowe, E.H., Briscoe, D.A., and Frankham, R. 2003. Fitness and adaptation in a novel environment: effect of inbreeding, prior environment, and lineage. Evolution 57:1822-1828.

Retamal, R., Zaviezo, T., Malausa, T., Fauvergue, X., Le Goff, I., and Toleubayev, K. 2016. Genetic analyses and occurrence of diploid males in field and laboratory populations of Mastrus ridens (Hymenoptera: Ichneumonidae), a parasitoid of the codling moth. Biological Control 101:69-77.

Sandanayaka, W.R.M., Chhagan, A., Page-Weir, N.E.M., and Charles, J.G. 2011a. Colony optimization of Mastrus ridens (Hymenoptera: Ichneumonidae), a potential biological control agent of codling moth in New Zealand. New Zealand Plant Protection 64:227-234.

Sandanayaka, W.R.M., Chhagan, A., Page-Weir, N.E.M., de Silva, H.H., and Charles, J.G. 2011b. The effect of mating behaviour on progeny sex ratio of Mastrus ridens (Hymenoptera: Ichneumonidae), a biological control agent of codling moth Biocontrol Science and Technology 21:485-496.

Taylor, S.J., Downie, D.A., and Paterson, I.D. 2011. Genetic diversity of introduced populations of the water hyacinth biological control agent Eccritotarsus catarinensis (Hemiptera: Miridae). Biological Control 58:330-336.

Tortosa, O.E., Carmona, A., Martínez, E., Manzano, P., y Giardina, M. 2014. Liberación y establecimiento de Mastrus ridens (Hymenoptera: Ichneumonidae) para el control de Cydia pomonella (Lepidoptera: Tortricidae) en Mendoza Argentina. Revista de la Sociedad Entomológica Argentina 73:109-118.

van Baalen, M., and Hemerik, L. 2008. Parasitoid fitness: from a simple idea to an intricate concept. p. 31-50. In Wajnberg, E., Bernstein, C., and van Alphen, J. (eds.) Behavioral ecology of insect parasitoids: From theoretical approaches to field applications. Blackwell Publishing, Malden, Massachusetts, USA.

van Driesche, R., Hoddle, M., and Center, T. 2009. Control of pests and weeds by natural enemies: An introduction to biological control. John Wiley and Sons, Hoboken, New Jersey, USA.

van Lenteren, J.C. 2012. The state of commercial augmentative biological control: Plenty of natural enemies, but a frustrating lack of uptake. Biocontrol 57:1-20. 
Wajnberg, E., Bernstein, C., and van Alphen, J. 2008. Behavioral ecology of insect parasitoids: From theoretical approaches to field applications. 445 p. Blackwell Publishing, Malden, Massachusetts, USA.

Woodworth, L.M., Montgomery, M.E., Briscoe, D.A., and Frankham, R. 2002. Rapid genetic deterioration in captive populations: causes and conservation implications. Conservation Genetics 3:277-288.
Zayed, A., and Packer, L. 2005. Complementary sex determination substantially increases extinction proneness of haplodiploid populations. Proceedings of the National Academy of Sciences of the United States of America 102:10742-10746. 